\title{
Factors Facilitating Fusion between Dye-encapsulating Vesicles and Giant Unilamellar Vesicles
}

\author{
Daiya Mombayashi, Ami Yasuhara, Kai Hashino, \\ Akira Heya, and Koji Sumitomo* \\ Department of Materials and Synchrotron Radiation Engineering, University of Hyogo, \\ 2167 Shosha, Himeji, Hyogo 671-2280, Japan
}

(Received June 8, 2021; accepted October 13, 2021)

Keywords: vesicle fusion, molecular transport, dye-encapsulating vesicles, nanobiotechnology, flow control system

Vesicle fusion plays an important role in molecular transport and signal transduction in vivo. Fusion occurs even in artificial vesicles without proteins, as long as cations are present similar to the physiological environment. The progress of fusion between vesicles encapsulating a fluorescent dye (calcein) and giant unilamellar vesicles (GUVs) was examined using a fluorescence microscope. In the case of vesicles formed with phosphatidylcholine (PC) alone, the fusion begins upon the suppression of electrostatic repulsion by cations such as $\mathrm{Ca}^{2+}$. However, almost all vesicles remain in the state of adsorption or hemifusion even after $1 \mathrm{~h}$ or more, and it is difficult to proceed to full fusion. In contrast, mixing phosphatidylethanolamine (PE) with vesicles facilitates the progression from hemifusion to full fusion, and the transfer of calcein to the GUV was observed immediately after beginning the vesicle fusion. This is probably because the small head group makes the fluid phase lipid bilayer unstable.

\section{Introduction}

Vesicle fusion has been extensively studied because it plays an important role in molecular transport and signal transduction in vivo. In biological cells, vesicle fusion is usually triggered and controlled by membrane proteins, such as SNARE proteins ${ }^{(1-3)}$ and viral fusion proteins. ${ }^{(4,5)}$ To elucidate the mechanism of specific vesicle fusion in vivo, knowledge of the precise structures of membrane proteins and the interaction between membrane proteins and lipid bilayers is required. However, under some conditions, lipid membranes fuse even in the absence of membrane proteins. Protein-free membrane fusion is also important for understanding the detailed mechanism of vesicle fusion and has been extensively studied both experimentally and theoretically. ${ }^{(6-9)}$

On the other hand, approaches that combine artificial lipid membranes and nanostructures have been actively investigated for the development of devices for the functional measurement of membrane proteins and the detection of biomolecules. ${ }^{(10,11)}$ As a method of introducing a

*Corresponding author: e-mail: sumitomo@eng.u-hyogo.ac.jp

https://doi.org/10.18494/SAM.2021.3454 
membrane protein into a nanobiodevice, the fusion of proteoliposomes, which are vesicles in which membrane proteins are reconstituted, is a promising method. However, the fusion efficiency is not sufficiently high. The improvement and control of fusion efficiency are the hurdles to overcome for the fabrication of nanobiodevices.

In this study, we investigated the protein-free fusion of vesicles composed of 1,2-dioleoyl-snglycero-3-phosphocholine (DOPC), which is the main component of lipids in biological cell membranes. It is one of the most important steps in protein-induced membrane fusion in vivo. The fusion between dye-encapsulating vesicles and giant unilamellar vesicles (GUVs) was observed using a fluorescence microscope. To evaluate the factors facilitating fusion, the vesicle fusion was observed under various conditions, such as the lipid composition of vesicles and the ion concentration in the solution. The flow of the vesicle dispersion toward the GUVs was controlled and the fusion process was also observed.

\section{Materials and Methods}

\subsection{Materials}

DOPC, 1,2-dioleoyl-sn-glycero-3-phospho-L-serine (sodium salt) (DOPS), 1,2-dioleoyl-snglycero-3-phosphoethanolamine (DOPE), 1,2-dioleoyl-sn-glycero-3-ethylphosphocholine (chloride salt) (EDOPC), 1,2-dioleoyl-sn-glycero-3-phosphoethanolamine- $N$-(lissamine rhodamine B sulfonyl) (Rhod-DOPE), and 1,2-distearoyl-sn-glycero-3-phosphoethanolamine- $N$ [biotinyl(polyethylene glycol)-2000] [DSPE-PEG(2000) Biotin] were purchased from Avanti Polar Lipids (Alabaster AL). Bovine serum albumin (BSA) and biotin-labeled BSA were purchased from Sigma-Aldrich (St. Louis, MO). Bis[N,N-bis(carboxymethyl) aminomethyl] fluorescein (calcein) was purchased from MP Biomedicals (Japan). Sucrose, glucose, calcium chloride, and other chemicals were purchased from Fujifilm Wako (Japan). All chemicals were used as received without further purification.

\subsection{Preparation of dye-encapsulating vesicles}

Large unilamellar vesicles (LUVs) were prepared by extrusion. A chloroform solution of DOPC or a lipid mixture (DOPC/DOPS, DOPC/DOPE) including Rhod-DOPE (0.5 mol\%) for fluorescent labeling was dried in a glass vial and suspended in a calcein solution $(50 \mathrm{mM})$ to a lipid concentration of $1 \mathrm{mM}$. To increase the entrapment efficiency of the calcein solution, the lipid suspension was subjected to five freeze/thaw cycles by alternately placing the sample vial in a liquid $\mathrm{N}_{2}$ bath and a warm water bath. To obtain uniformly sized unilamellar vesicles, the vesicles were extruded with a mini-extruder (Avanti Polar Lipids) using a Nuclepore polycarbonate membrane of $100 \mathrm{~nm}$ pore size (Whatman, GE Healthcare UK Ltd.). Calcein not encapsulated in LUVs was removed by a spin column technique. Sephadex G-50 Superfine (Sigma-Aldrich) was used as a gel filtration medium, and the spin column was centrifuged at $2000 \mathrm{~g}$ for $45 \mathrm{~s}$. By repeating the gel filtration 10 times, a calcein-encapsulating LUV dispersion was prepared and immediately used for experiments. 


\subsection{Preparation of GUVs}

GUVs were prepared by the electroformation method ${ }^{(12)}$ as follows. The chloroform solution of DOPC or lipid mixture (DOPC/EDOPC, DOPC/DOPE) was applied as uniformly as possible to an ITO-coated glass substrate. Then, a sucrose solution $(230 \mathrm{mM})$ was sandwiched between it and a clean ITO-coated glass substrate at an interval of $1 \mathrm{~mm}$, and an alternating electric field was applied. The typical conditions were an $\mathrm{AC}$ voltage of $1 \mathrm{~V}$ at $10 \mathrm{~Hz}$ for $2 \mathrm{~h}$ at room temperature. It was fluorescently labeled for observation by mixing Rhod-DOPE $(0.5 \mathrm{~mol} \%)$ in the chloroform solution.

\subsection{Observation of fluorescence images}

GUVs before and after fusion with calcein-encapsulating LUVs were observed with an upright microscope BX53 (Olympus, Japan). Fluorescence filter cubes U-FBNA (EX470-495/ EM510-550/DN505) and U-FGW (EX530-550/EM575IF/DN570) were chosen for observing calcein and rhodamine, respectively. Fluorescence images were captured with a digital camera, EOS Kiss X9 (Canon, Japan), and analyzed with the processing software ImageJ (National Institutes of Health, MD). In the flow control system described below, fluorescence images were observed using the confocal laser scanning microscope FV3000/BX63 (Olympus, Japan). Lasers at 488 and $561 \mathrm{~nm}$ were used to excite calcein and rhodamine, respectively.

\subsection{Flow control of LUV to immobilized GUV on substrate}

To observe the fusion process, we applied a system in which a certain amount of LUV dispersion was allowed to flow toward the GUV immobilized on the substrate, as shown in Fig. 1 . The GUV was prepared by mixing $1 \%$ of biotin-functionalized lipids [DSPE-PEG(2000) Biotin]. The cover slip in the observation chamber with a capacity of $200 \mu \mathrm{L}$ was coated with BSA/biotinylated-BSA (99:1), and the GUVs were immobilized on the cover slip by biotin-

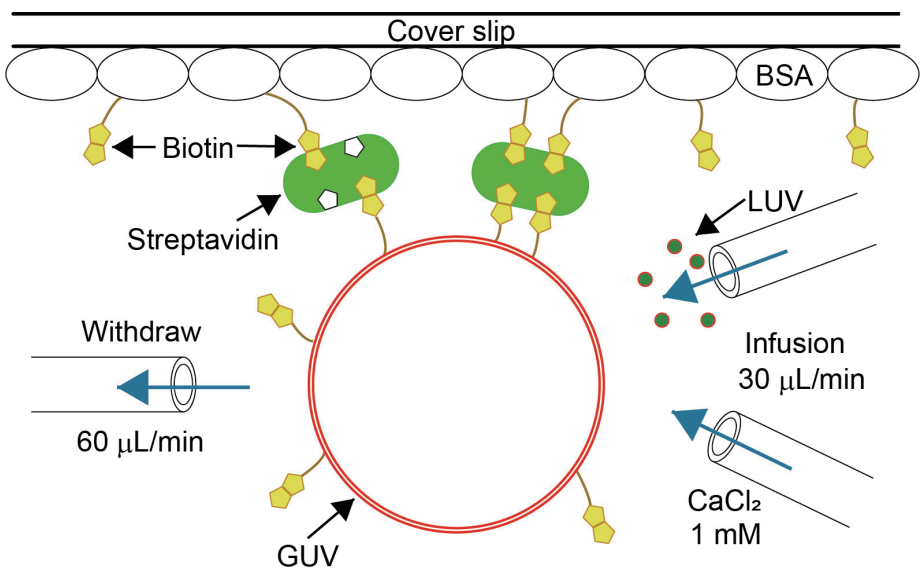

Fig. 1. (Color online) Flow control system. 
streptavidin interaction. ${ }^{(13,14)}$ Then, each glucose solution $(200 \mathrm{mM})$ containing calceinencapsulating LUVs and $1 \mathrm{mM} \mathrm{CaCl}_{2}$ was infused into the chamber at a rate of $30 \mu \mathrm{L} / \mathrm{min}$ using a syringe pump, Fusion 200 Touch (Chemyx, Stafford, TX). Simultaneously, the mixed solution was withdrawn at $60 \mu \mathrm{L} / \mathrm{min}$.

\section{Results and Discussion}

\subsection{Vesicle fusion by electrostatic attraction}

Electrostatic attraction is one of the most controllable interactions. Cationic lipids have been widely used as a means of transfection into biological cells, which are negatively charged. ${ }^{(15)}$ An efficient protein-free vesicle fusion between anionic GUVs and cationic vesicles has been reported..$^{(16,17)}$ The selective fusion of anionic LUVs to a supported lipid bilayer containing cationic lipids has also been reported. ${ }^{(18)}$ In this study, we observed the fusion of cationic GUVs and anionic LUVs. After mixing each dispersion in a microtube, they were immediately dropped to a glucose solution for fluorescence observation. Figure 2 shows the typical fluorescence images of a GUV and the intensity distribution of the green fluorescence of the 10 observed GUVs. The two dashed lines in Fig. 2(c) show the fluorescence intensity of GUVs containing 0.05 and $0.5 \mu \mathrm{M}$ calcein. The strong fluorescence from calcein observed inside the GUV indicates that the calcein-encapsulating LUVs were completely fused and the calcein was transferred inside the GUV. From the difference in calcein concentration $(50 \mathrm{mM})$ in LUVs and the volumes of GUVs and LUVs, it is estimated that 100 to 1000 LUVs were fused. It was confirmed that many LUVs were immediately and completely fused by electrostatic attraction.

\subsection{Vesicle fusion promoted by $\mathrm{Ca}^{2+}$ ions}

Next, we investigated the fusion of GUVs and LUVs, which consist of electrically neutral lipids (DOPC). Figures 3(a) and 3(b) show the fluorescence images of a GUV incubated in a 230 $\mathrm{mM}$ glucose solution for $1 \mathrm{~h}$ after mixing. No calcein fluorescence was observed inside the

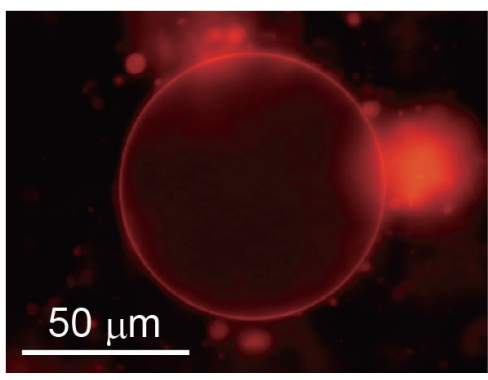

(a)

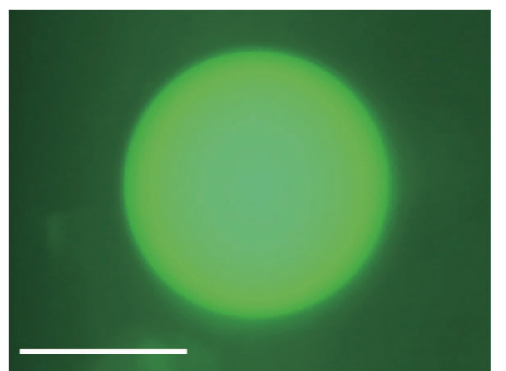

(b)

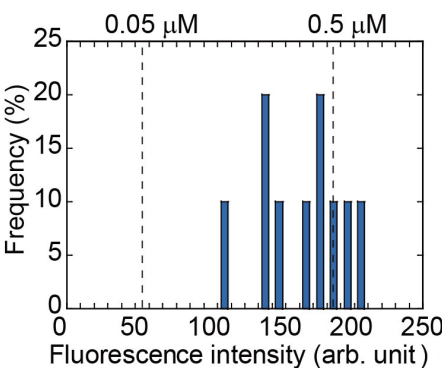

(c)

Fig. 2. (Color online) Fluorescence microscopy images obtained immediately after mixing cationic GUV and anionic LUV. Fluorescence from (a) rhodamine and (b) calcein. (c) Distribution of calcein fluorescence intensity. 


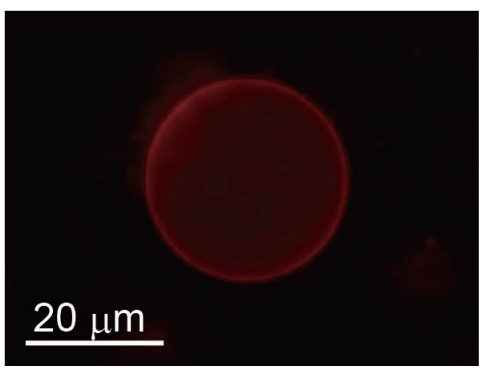

(a)

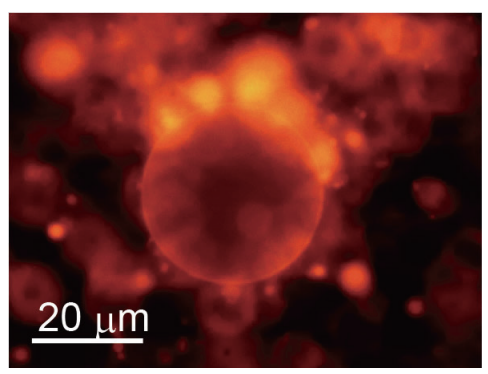

(d)

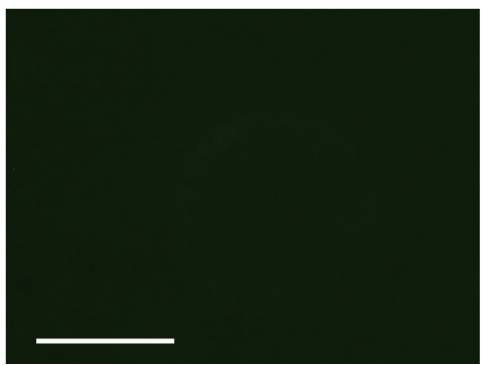

(b)

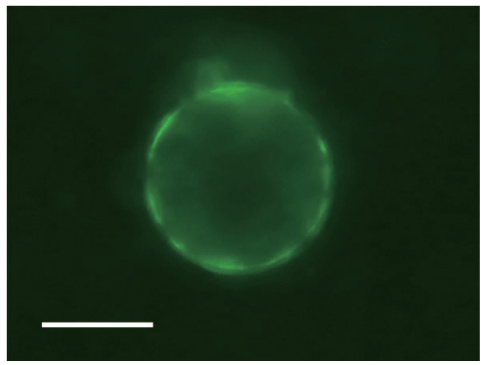

(e)

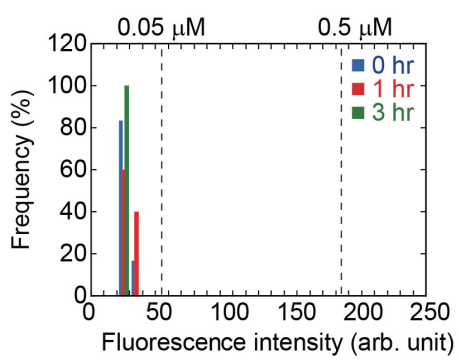

(c)

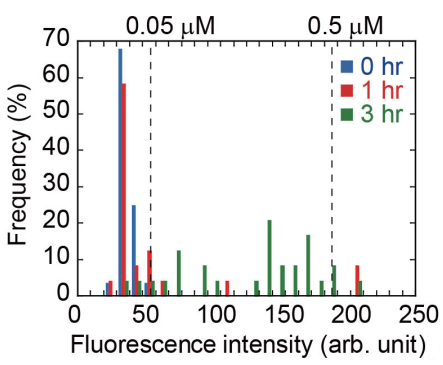

(f)

Fig. 3. (Color online) Fluorescence images of GUV incubated for $1 \mathrm{~h}$ after mixing with LUV in solution (a), (b) with and (d), (e) without $\mathrm{Ca}^{2+}$ ions. Distribution of calcein fluorescence intensity (c) with and (f) without $\mathrm{Ca}^{2+}$ ions.

GUV, indicating that fusion did not occur. The intensity of green fluorescence did not change from the background level even when the incubation time was extended, as shown in Fig. 3(c).

On the other hand, in some GUVs incubated in a glucose solution containing $1 \mathrm{mM} \mathrm{CaCl}_{2}$, calcein fluorescence was observed, as shown in Figs. 3(d) and 3(e). The progress of fusion is considered to depend on the concentration of $\mathrm{CaCl}_{2}$; but this time, the experiment was conducted under the typical physiological condition (extracellular) of $1 \mathrm{mM}$. The aggregation of GUVs has also become remarkable. After incubation for $3 \mathrm{~h}$, the fluorescence intensity distribution of calcein shifted to the brighter side, indicating that the fusion progressed with elapsed time.

Although the phosphatidylcholine (PC) group is zwitterionic and thus uncharged at neutral $\mathrm{pH}$, the surface potential of the vesicle consisting of DOPC is slightly negative. ${ }^{(19)}$ The weak negative zeta potential has been interpreted in terms of the orientation of the hydration layers and lipid head groups. In addition, since the lipid labeled with the fluorescent dye (Rhod-DOPE) is negatively charged, the negative surface charge of the vesicle increases, although the composition ratio is as small as $0.5 \mathrm{~mol} \%$. The zeta potential of the DOPC LUV used in this experiment was $-10.6 \pm 2.0 \mathrm{mV}$ (mean $\pm \mathrm{SD}$ ). Vesicles with a negatively charged surface never fuse even when incubated for a long time owing to electrostatic repulsion when cations such as $\mathrm{Ca}^{2+}$ are not present in the solution. When $\mathrm{CaCl}_{2}$ is added to a solution, $\mathrm{Ca}^{2+}$ acts like a bridge between the negatively charged vesicles, causing fusion. This is the same as the formation of a supported lipid bilayer by the vesicle fusion method on a negatively charged substrate such as $\mathrm{SiO}_{2}{ }^{(20)}$ It is shown that the divalent cation $\left(\mathrm{Ca}^{2+}\right)$ promotes fusion. 


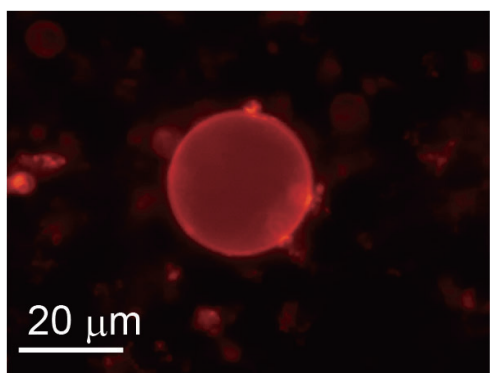

(a)

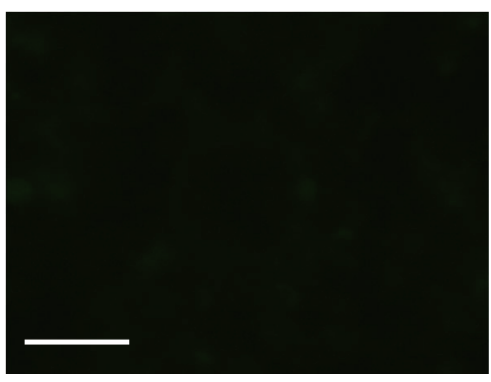

(b)

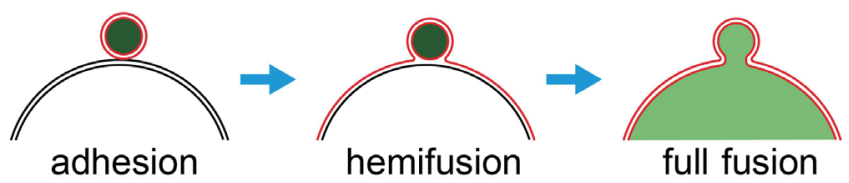

(c)

Fig. 4. (Color online) Fluorescence images of an unlabeled GUV incubated for $1 \mathrm{~h}$ after mixing with calceinencapsulating LUV labeled with rhodamine. Fluorescence from (a) rhodamine and (b) calcein. (c) Schematic illustration of each step of vesicle fusion.

To investigate the fusion process, unlabeled GUVs and calcein-encapsulating LUVs were mixed. LUV lipids include rhodamine-labeled lipids. As shown in Fig. 3(f), $\mathrm{Ca}^{2+}$ induces vesicle fusion, but there are many GUVs in which the fluorescence intensity of calcein has not yet increased (remains at the background level) even after $1 \mathrm{~h}$ of incubation. Rhodamine fluorescence has also been observed in such GUVs [Fig. 4(a)]. In this case, since the GUV does not contain fluorescent-labeled lipids, the fluorescence of rhodamine is due to the adhesion or fusion of LUVs. Vesicle fusion proceeds to complete the fusion through a hemifusion state after adhesion, as shown in Fig. 4(c). ${ }^{(7)}$ Since the lipids in the outer leaflet of the LUV are fused with the GUV even in the hemifusion state, the lipids labeled with rhodamine move to the GUV. On the other hand, the contained calcein does not move to the GUV. Furthermore, since the calcein concentration is high $(50 \mathrm{mM})$ in the LUV, the fluorescence intensity does not increase considerably because of the self-quenching effect. There is an energy barrier between hemifusion and full fusion, indicating that many remain in the hemifusion state even after fusion is induced by $\mathrm{Ca}^{2+}$.

\subsection{Promotion of vesicle fusion by mixing DOPE}

The promotion of vesicle fusion by the structure of lipid molecules was investigated by mixing phosphatidylethanolamine (PE). ${ }^{(21-23)}$ While PC has a cylindrical shape, PE has a cone shape owing to the difference in the head group. While PC forms a stable lipid bilayer, PE does not owing to the negative curvature caused by its shape. When the PE content is high, hexagonal phase structures are formed instead of lipid bilayer membranes. ${ }^{(23)}$ Here, DOPC and DOPE were mixed at a ratio of 7:3 to prepare the LUV for investigating vesicle fusion with GUVs consisting 


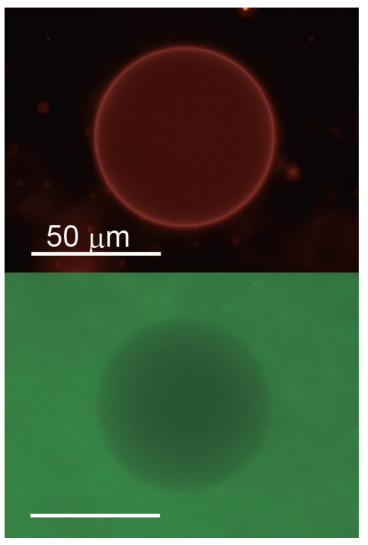

(a)

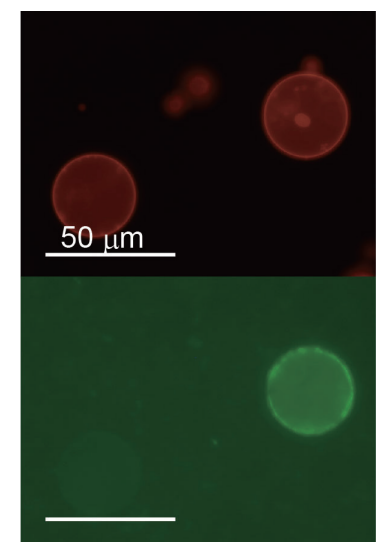

(b)

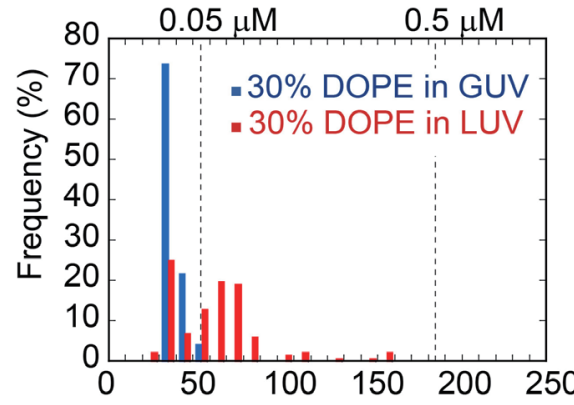

Fluorescent intensity (arb. units)

(c)

Fig. 5. (Color online) (a) Fluorescence microscopy images obtained immediately after mixing GUV and LUV consisting of DOPC/DOPE mixture. (b) After solution replacement. (c) Distribution of calcein fluorescence intensity in GUVs.

of DOPC. As shown in Fig. 5(a), the LUV was unstable in a solution containing $1 \mathrm{mM} \mathrm{Ca}^{2+}$ ions, and the leakage of calcein from the LUV was immediately observed. When the GUV was separated by soft centrifugation at $2000 \mathrm{~g}$ and the solution was replaced, it was found that calcein was also transferred to the GUV by vesicle fusion as shown in Fig. 5(b). It is considered that the inclusion of DOPE in the lipid mixture promoted the fusion of LUVs and many calcein leaks were observed. At the same time, fusion with the GUV is also promoted. When DOPE was mixed with the GUV instead of the LUV, vesicle fusion was promoted. Figure 5(c) shows the distribution of the fluorescence intensity of calcein transferred to the GUV by vesicle fusion in each case. It can be seen that the fusion-promoting effect becomes greater when DOPE is mixed with the LUV. This is probably because the negative curvature effect of DOPE becomes more remarkable when DOPE is mixed with the LUV with a large curvature. The fact that curvature has a significant effect on the progression of fusion appears to be strongly related to the fact that vesicle fusion in vivo involves the deformation of the lipid membrane.

\subsection{Time lapse imaging during vesicle fusion}

To observe the fusion process, the GUVs were immobilized on the coverslip, and time-lapse observation was performed during vesicle fusion. LUVs prepared with a DOPC/DOPE (7:3) mixture were used to promote fusion. The LUV dispersion was diluted 100-fold with a glucose solution and infused into the observation chamber by a syringe pump. To avoid the fusion of LUVs, a $\mathrm{CaCl}_{2}$ solution $(1 \mathrm{mM})$ was introduced separately and mixed with the LUV dispersion in the observation chamber. All solutions were adjusted to have the same osmotic pressure with glucose. Figures 6(a) and 6(b) show typical GUV fluorescence microscopy images obtained before and $780 \mathrm{~s}$ after mixing the LUVs. The immobilization of the GUVs on the cover slip via biotin-streptavidin interactions allowed the continuous observation of the target GUVs. The 


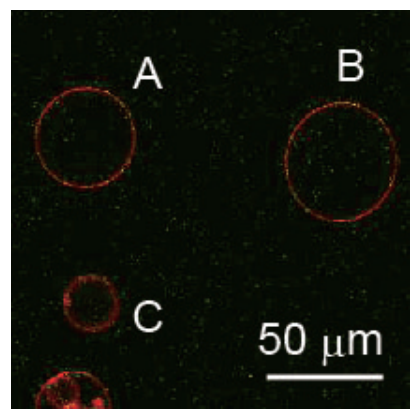

(a)

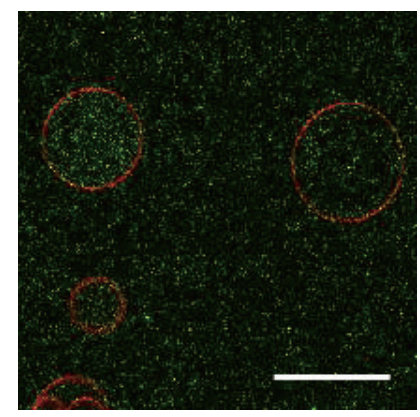

(b)

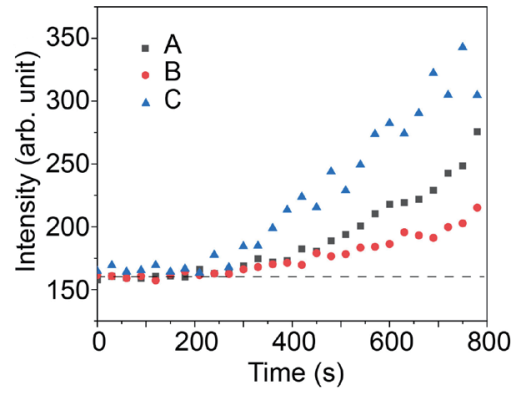

(c)

Fig. 6. (Color online) Typical GUV fluorescence microscopy images obtained (a) before and (b) $780 \mathrm{~s}$ after mixing LUVs. (c) Changes in calcein fluorescence intensity over time.

fluorescence of calcein in the GUVs increased from the background level before fusion after the infusion of each glucose solution containing LUVs and $\mathrm{CaCl}_{2}$. Although the fusion efficiency differs depending on the GUV, Fig. 6(c) indicates that the calcein fluorescence intensity increased and the fusion progressed with elapsed time. It was confirmed that the LUV consisting of a DOPC/DOPE mixture easily fuses with the GUV in the solution containing $\mathrm{Ca}^{2+}$ and immediately progresses to the full-fusion state owing to its structural instability.

\section{Conclusions}

Vesicle fusion between GUVs and dye-encapsulating LUVs was investigated using a fluorescence microscope. Vesicles containing even only PC can cause vesicle fusion by suppressing the electrostatic repulsion caused by cations such as $\mathrm{Ca}^{2+}$. However, even after $1 \mathrm{~h}$ or more, most of them remain in the state of adsorption and hemifusion and do not progress to the state of full fusion. In contrast, vesicles containing PE, whose small head group destabilizes the fluid phase lipid bilayer, facilitate the process from hemifusion to full fusion. The immobilization of the GUV on the cover slip and the flow control of the LUV dispersion toward the GUV enabled the time-lapse imaging of the vesicle fusion in the targeted GUV. The curvature-dependent progression of vesicle fusion is also associated with the deformation of the lipid membrane in vivo. Protein-free membrane fusion is also an important process in proteininduced fusion, and this finding helps us understand vesicle fusion in vivo. Understanding and facilitating vesicle fusion will also contribute to the development of nanobiodevices by controlling the fusion of proteoliposomes.

\section{References}

1 T. Weber, B. V. Zemelman, J. A. McNew, B. Westermann, M. Gmachl, F. Parlati, T. H. Söllner, and J. E. Rothman: Cell 92 (1998) 759. https://doi.org/10.1016/S0092-8674(00)81404-X

2 J. S. Bonifacino and B. S. Glick: Cell 116 (2004) 153. https://doi.org/10.1016/S0092-8674(03)01079-1

3 J. Rizo: Protein Sci. 27 (2018) 1364. https://doi.org/10.1002/pro.3445

4 K. L. Hefferon, A. G. P. Oomens, S. A. Monsma, C. M. Finnerty, and G. W. Blissard: Virology 258 (1999) 455. https://doi.org/10.1006/viro.1999.9758 
5 K. Kamiya, J. Kobayashi, T. Yoshimura, and K. Tsumoto: Biochim. Biophys. Acta, Biomembr. 1798 (2010) 1625. https://doi.org/10.1016/j.bbamem.2010.05.011

6 L. V. Chernomordik and M. M. Kozlov: Nat. Struct. Mol. Biol. 15 (2008) 675. https://doi.org/10.1038/nsmb.1455

7 R. P. Stock, J. Brewer, K. Wagner, B. Ramos-Cerrillo, L. Duelund, K. D. Jenshøj, L. F. Olsen, and L. A. Bagatolli: PLoS One 7 (2012) e36003. https://doi.org/10.1371/journal.pone.0036003

8 R. Fraley, J. Wilschut, N. Düzgüneş, C. Smith, and D. Papahadjopoulos: Biochemistry 19 (1980) 6021. https:// doi.org/10.1021/bi00567a012

9 S. Kawamoto, M. L. Klein, and W. Shinoda: J. Chem. Phys. 143 (2015) 243112. https://doi.org/10.1063/1.4933087

10 K. Sumitomo, A. McAllister, Y. Tamba, Y. Kashimura, A. Tanaka, Y. Shinozaki, and K. Torimitsu: Biosens. Bioelectron. 31 (2012) 445. https://doi.org/10.1016/j.bios.2011.11.010

11 R. Watanabe, N. Soga, D. Fujita, K. V. Tabata, L. Yamauchi, S. H. Kim, D. Asanuma, M. Kamiya, Y. Urano, H. Suga, and H. Noji: Nature Commun. 5 (2014) 4519. https://doi.org/10.1038/ncomms5519

12 F. M. Menger and M. I. Angelova: Acc. Chem. Res. 31 (1998) 789. https://doi.org/10.1021/ar970103v

13 A. R. Patel and C. W. Frank: Langmuir 22 (2006) 7587. https://doi.org/10.1021/la0610452

14 D. Stamou, C. Duschl, E. Delamarche, and H. Vogel: Angew. Chem. Int. Ed. 42 (2003) 5580. https://doi. org/10.1002/anie.200351866

15 J. Zabner, Al J. Fasbender, T. Moniger, K. A. Poellinger, and M. J. Welsh: J. Biol. Chem. 270 (2995) 18997. https://doi.org/10.1074/jbc.270.32.18997

16 R. B. Lira, T. Robinson, R. Dimova, and K. A. Riske: Biophys. J. 116 (2019) 79. https://doi.org/10.1016/j. bpj.2018.11.3128

17 R. R. Ishmukhametov, A. N. Russell, and R. M. Berry: Nature Commun. 7 (2016) 13025. https://doi. org/10.1038/ncomms13025

18 A. Oshima and K. Sumitomo: Biochem. Biophys. Rep. 11 (2017) 58. https://doi.org/10.1016/j.bbrep.2017.06.003

19 B. Klasczyk, V. Knecht, R. Lipowsky, and R. Dimova: Langmuir 26 (2010) 18951. https://doi.org/10.1021/ la103631y

20 R. Richter, A. Mukhopadhyay, and A. Brisson: Biophys. J. 85 (2003) 3035. https://doi.org/10.1016/S00063495(03)74722-5

21 M. J. Hope, D. C. Walker, and P. R. Cullis: Biochem. Biophys. Res. Commun. 110 (1983) 15. https://doi. org/10.1016/0006-291X(83)91253-6

22 J. W. Holland, C. Hui, P. R. Cullis, and T. D. Madden: Biochemistry 35 (1996) 2618. https://doi.org/10.1021/ bi952000v

23 A. M. Sendecki, M. F. Poyton, A. J. Baxter, T. Yang, and P. S. Cremer: Langmuir 33 (2017) 13423. https://doi. org/10.1021/acs.langmuir.7b02323 